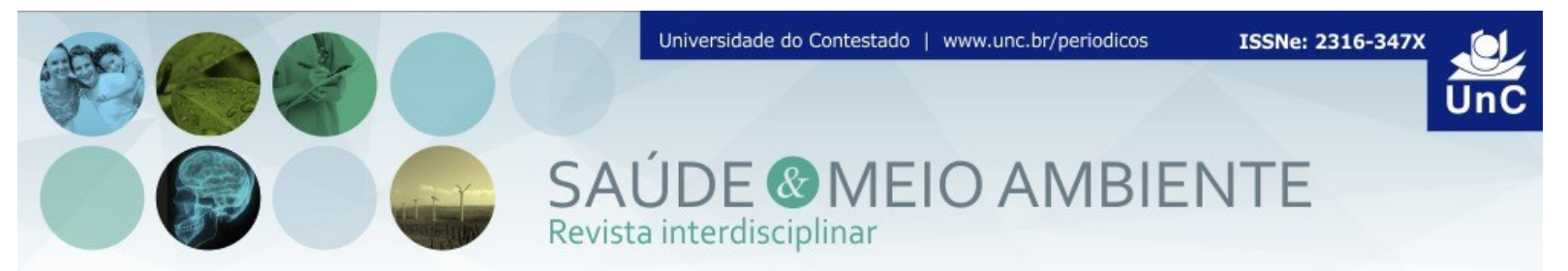

\title{
DISPONIBILIDADE DE MEDICAMENTOS TÓPICOS E OCULARES PARA O TRATAMENTO DE CONJUNTIVITE ALÉRGICA NAS FARMÁCIAS PRIVADAS DA CIDADE DE NAMPULA, MOÇAMBIQUE, 2019
}

\author{
AVAILABILITY OF TOPICAL AND OCULAR MEDICATIONS FOR THE \\ TREATMENT OF ALLERGIC CONJUNCTIVITIS IN PRIVATE PHARMACIES IN \\ NAMPULA CITY, MOZAMBIQUE, 2019
}

Joel de Melo Bambamba ${ }^{1}$

Ângela Joana Efécio ${ }^{2}$

Belmiro Raúl Constantino ${ }^{3}$ Jorge Josaphat Ferreira ${ }^{4}$

\begin{abstract}
RESUMO
A Conjuntivite é um processo inflamatório da conjuntiva, caracterizado pela dilatação vascular, infiltração celular e exsudação. As patologias conjuntivais constituem 30 a $90 \%$ das consultas realizadas em oftalmología. Dentre elas as mais comuns são as patologias inflamatórias (conjuntivitis). A importância clínica da alergia ocular é devida mais a sua frequência do que a sua gravidade, apesar de poder levar a cegueira em alguns casos. Este estudo teve como objectivo, Avaliar a disponibilidade dos medicamentos tópico e oculares para o tratamento da conjuntivite alérgica nas Farmácias de cidade Nampula em Moçambique. Realizou-se um estudo observacional, dscritivo quantitativo, transversal e prospectivo, com base em um questionário para o levantamento da presença de 23 medicamentos. Das 41 farmácias, $87,8 \%$ tinham pelo menos 1 medicamento dos 23 nomes que constavam no questionário. Dos 23 itens seleccionados foram encontrados 13, correspondentes a $54,52 \%$, a predniselona foi o mais encontrado com $82.9 \%$, a Dexametazona com $21.6 \%$. O grupo mais disponível foi de Corticosteroides $82,9 \%$, seguido de Estabilizadores de mastócitos com $46,3 \%$ e o grupo indisponível foi o de antihistamínicos. De forma geral, a disponibilidade de medicamentos para tratamento da conjuntivite alérgica nas farmácias da cidade de Nampula é relativamente alta, um
\end{abstract}

\footnotetext{
${ }^{1}$ Mestrando em Direcção Estrategica para Organizações de Saúde com especialidade em Gestão e pesquisa Sanitária da Fundação IberAmericana - UNINI Porto Rico, Licenciado em Optometria pela Universidade Lúrio. Docente no curso de Licenciatura em Optometria na Universidade Lúrio em Moçambique. Campus de Marrere. Nampula, Moçambique. E- mail: joelmbambamba@gmail.com

${ }^{2}$ Mestre em Optometria Clínica e Visão pela Universidade de Alicante. Licenciada em Optometria pela Universidade Lúrio. Docente do curso de Licenciatura em Optometria na Universidade Lúrio em Moçambique. Campus de Marrere. Nampula, Moçambique. E-mail: angy.joana@gmail.com

3 Estudante do Curso de Licenciatura em Optometria na Universidade Lúrio em Moçambique. Campus de Marrere, Nampula, Moçambique. E-mail: belmiro19921@gmail.com

${ }^{4}$ Mestre em Saúde Pública pela Faculdade de Medicina da Universidade Federal de Minas. Mestre em Epidemiologia pela faculdade de Medicina da Universidade do Porto. Docente na Universidade Única de Contagem (ISEIB) de Belo Horizonte. Belo Horizonte. Minas Gerais. Brasil. E-mail: jorgejosap@gmail.com
} 
número significativo de medicamentos está disponível nas farmácias privadas da cidade de Nampula, no entanto, verificou-se a ausência dos anti-histamínicos.

Palavras Chaves: Disponibilidade. Medicamento ocular. Tratamento. Conjuntivite Alérgica. Corticosteroides. Estabilizadores de mastócitos. Anti-histamínicos.

\begin{abstract}
Conjunctivitis is an inflammatory process of the conjunctiva, characterized by vascular dilation, cell infiltration and exudation. Conjunctive pathologies make up 30 to $90 \%$ of consultations in ophthalmology. Among them, the most common are inflammatory pathologies (conjunctivitis). The clinical importance of ocular allergy is due to its frequency rather than its severity, although it can lead to blindness in some cases. This study aimed to assess the availability of topical and eye medications for the treatment of allergic conjunctivitis in pharmacies in Nampula city in Mozambique. An observational, descriptive quantitative, cross-sectional and prospective study was conducted, based on a questionnaire to survey the presence of 23 drugs. Of the 41 pharmacies, $87.8 \%$ had at least $1 \mathrm{drug}$ from the 23 names on the questionnaire. Of the 23 selected items, 13 were found, corresponding to $54.2 \%$, prednisone was the most found with $82.9 \%$, Dexamethasone with $21.6 \%$. The most available group was Corticosteroids $82.9 \%$, followed by mast cell stabilizers with $46.3 \%$ and the unavailable group was antihistamines. In general, the availability of drugs for the treatment of allergic conjunctivitis in pharmacies in the city of Nampula is relatively high, a significant number of drugs for the treatment of allergic conjunctivitis are available in private pharmacies in the city of Nampula, however, there was an absence of antihistamines.
\end{abstract}

Keywords: Availability. Eye medication. Treatment. Allergic Conjunctivitis. Corticosteroids. Mast cell stabilizers. Antihistamines.

\title{
INTRODUÇÃO
}

Moçambique é um pais com uma vasta área de $801509 \mathrm{~km}^{2}$, com uma população de 27 milhões de habitants. O pais é dividio em 11 provincias e, a Provincia de Nampula tem maior número de habitantes (5 milhões), com a maior parte da população em área rural (1).

De acordo com o Banco Mundial, Moçambique é um pais pobre com o produto Interno Bruto (PIB) estimado em 15,20 bilhões de Dolares Americanos (USD) em 2019 (2). 
Figura 1 - Municipio da cidade de Nampula, área em que a pesquisa foi desenvolvida.

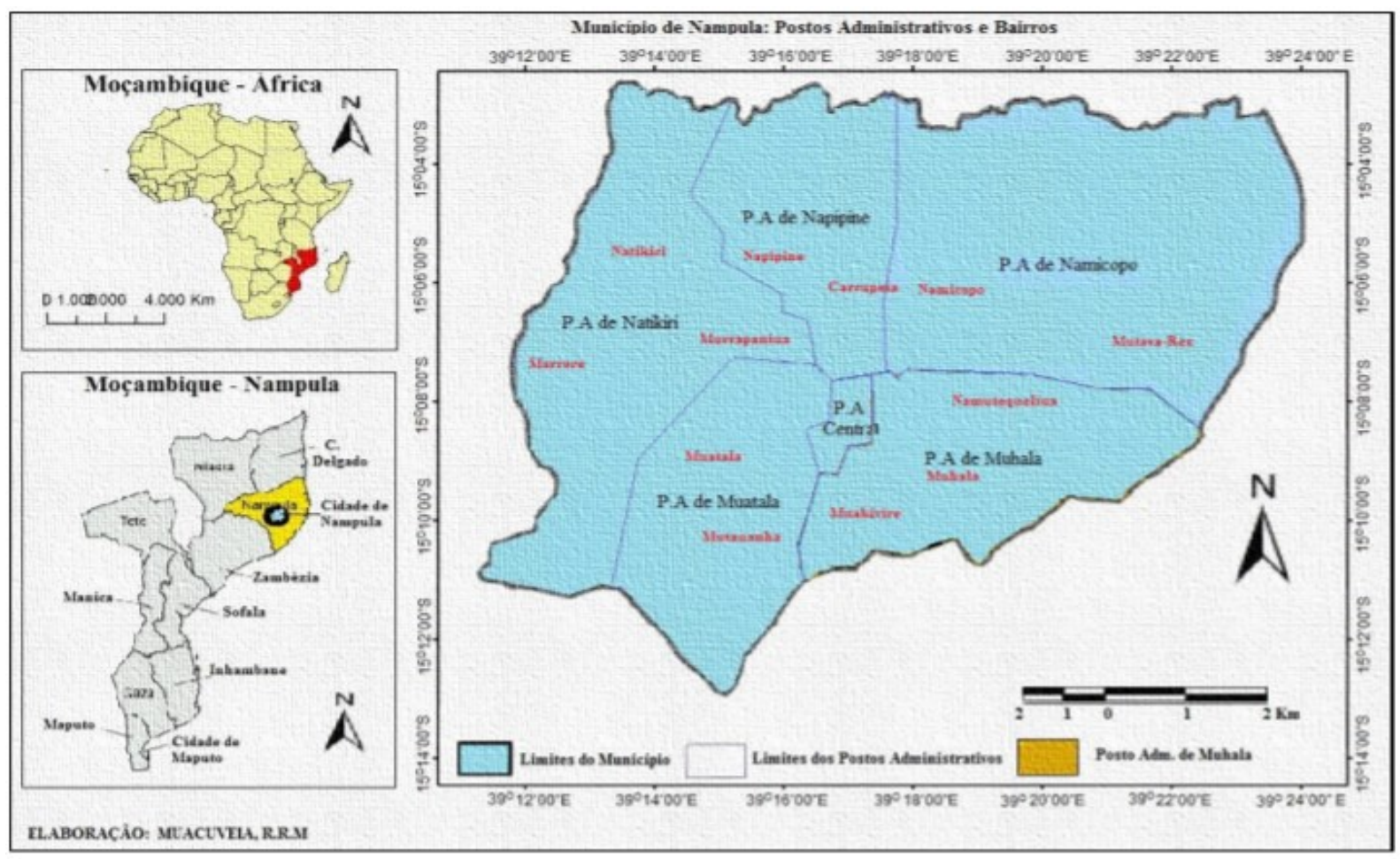

Fonte: Mucaveia (2017)

Nos últimos 30 anos, existiu uma redução na proporção de pessoas com deficiência visual e cegueira em todo o mundo. Contudo, populações em crescimento e envelhecimento significam que o desáfio de eliminar a cegueira evitável é agora maior ${ }^{(3)}$.

A prevenção da cegueira tem sido uma prioridade para a Organização Mundial da Saúde (OMS) por quase vinte anos através de seu programa de parceria com aAgência Internacional de Prevenção da Cegueira (IAPB) na VISÃO 2020. A principal premissa por trás dessa iniciativa é que até $80 \%$ da cegueira é evitável, isto é, tratável ou prevenivel, se existirem serviços disponíveis ${ }^{(4)}$.

A deficiência visual e a cegueira são preocupações de saúde a nível mundial e afetam 405 milhões e 36 milhões de pessoas, respectivamente. As comunidades e países mais afetados são aqueles com baixa acessibilidade a serviços e recursos inadequados ${ }^{(5)}$.

A conjuntivite, inflamação da conjuntiva que normalmente afeta estruturas adjacentes como a córnea, as vias lacrimais e as pálpebras, é uma doença muito comum em adultos e crianças. A resposta inflamatória dessa membrana pode ser causada por agentes infecciosos, tais como bactérias, vírus, fungos e parasitas, ou por agentes não infecciosos, tais como substâncias químicas, lentes de contato e alergia $^{(6)}$. 
Dados sobre a prevalência e as causas de deficiência visual (DV) e cegueira são necessários para planejar programas de eliminação da cegueira evitável e DV. Cerca de $90 \%$ dos indivíduos com deficiência visual do mundo vivem em países em desenvolvimento ${ }^{(7)}$.

Para maximizar as potencialidades das pessoas com DV é importante o desenvolvimento de recursos adaptados que potencializem o uso dos sentidos remanescentes, provendo acesso às informações ${ }^{(8)}$.

A Alergia ocular (AO) é um termo geral para descrever diferentes fenótipos, com conjuntivite alérgica sazonal e perene representando a maioria dos diagnósticos. Várias doenças, como ceratoconjuntivite atópica e ceratoconjuntivite primaveril, afetam um pequeno número de pacientes ${ }^{(9)}$.

No continente Africano, principalmente na África subsariana, a ceratoconjuntivite vernal (CCV) é um problema de saúde pública muito frequente, embora associações entre a renite e a conjuntivite alérgica não tenham sido muito frequentes $14,15 \%{ }^{(10)}$.

De um modo geral, observa-se alta prevalência (20\%) sendo responsável pela diminuição na qualidade de vida em um número expressivo de pacientes, seja pelo incômodo dos sintomas ou pelas complicações advindas da mesma, além de ser uma doença subdiagnosticada, sendo frequente a automedicação Estudos demonstram que poucos pacientes são avaliados por especialistas oftalmologistas e/ou alergistas. Entretanto, casos graves (2\%) geralmente procuram serviços especializados, o que pode ser responsável pela alta prevalência destes em alguns estudos ${ }^{(11)}$.

O acesso a medicamentos é um componente indispensável para que as populações tenham uma cobertura universal e equânime de saúde, com resolubilidade e qualidade, sendo reconhecido pela Organização das Nações Unidas (ONU) como um dos cinco indicadores relacionados a avanços na garantia do direito à saúde ${ }^{(12)}$.

O sector da saúde é um dos "sectores prioritários do Plano de acção para redução da Pobreza (PARP) em Moçambique, no entanto, testemunha-se nos últimos anos, uma ruptura constante de stock de medicamentos essenciais nas unidades sanitárias. Esta situação tem sido reportada nas várias regiões do país e por várias organizações da sociedade civil. (13).

Pesquisas realizadas em países de baixa renda corroboram que os medicamentos são escassos no sector público e inacessíveis nas farmácias privadas devido aos elevados preços praticados ${ }^{(12)}$.

Garantir o acesso aos cuidados em tempo oportuno é uma das características de sistemas de saúde de alta qualidade, mas alcançá-la é um problema em muitos países ${ }^{(14)}$.

O acesso a medicamentos tem sido compreendido a partir do equilíbrio entre quatro dimensões - disponibilidade, capacidade aquisitiva, aceitabilidade e acessibilidade geográfica, cujas barreiras se apresentam tanto na perspectiva da 
oferta quanto da demanda sendo as duas primeiras mais frequentemente abordadas nas medidas de acesso no nível internacional ${ }^{(15)}$.

Dentre as estratégias para a promoção do acesso da população a medicamentos, encontra-se a política de medicamentos genéricos. Em geral os genéricos possuem preços menores em relação aos medicamentos inovadores uma vez que não recaem sobre os genéricos, os custos relativos ao desenvolvimento da nova molécula e dos estudos clínicos ${ }^{(16)}$.

O Plano de Ação Global 2014-2019 foi adotado pelos estados-membros da Organização Mundial de saúde (OMS) na assembleia mundial de saúde em 2013. Seus objetivos são reduzir a deficiência visual como um problema de saúde pública global e assegurar o acesso à reabilitação para pessoas com deficiência visual (17).

Os medicamentos são muitas vezes o mais importante fator de custo dos gastos com saúde nos hospitais e cuidado ambulatório. Os pacientes que têm acesso a medicamentos adequados e eficazes no momento da necessidade são mais propensos a ficar felizes com o tratamento que recebem. Quando tais medicamentos não estão disponíveis ou ineficazes após o uso, os pacientes irão para outro lugar, mesmo que tenham que pagar preços elevados para provedores, para obter os cuidados que eles acham que precisam ${ }^{(18)}$.

Os países em desenvolvimento geralmente oferecem proteção menos substancial para patentes do que países industrializados. Muitos países em desenvolvimento tradicionalmente excluíram produtos farmacêuticos de proteção de patentes; apenas processos para a produção de produtos farmacêuticos poderia ser patenteado ${ }^{(19)}$.

A baixa disponibilidade de medicamentos na lista Nacional de Medicamentos Essenciais, no Formulário de medicamentos Nacional e em hospitais governamentais podem ter implicações diretas no acesso, uma vez que os pacientes precisam comprar esses medicamentos em farmácias privadas.

As restrições com que se deparam tanto os clínicos como os pacientes no acesso a medicamentos de eleição para o tratamento adequado de determindas doeças ainda é muito presente, principalmente em paises em processo de desenvolvimento.

Reconhecendo o impacto negativo que tem a conjuntivite alérgica na vida das pessoas e a importância da disponibilidade do medicamento como parte fundamental do processo de aquisição e consequente melhoria de qualidade de vida da população, é essencial, Avaliar a disponibilidade de medicamentos tópico e oculares para o tratamento de conjuntivite alérgica; identificar a existência de medicamentos de uso tópico e ocular para o tratamento da conjuntivite alérgica; e a posterior; determinar a disponibilidade dos medicamentos. 


\section{MATERIAIS E MÉTODOS}

Tratou-se de um estudo Transversal descritivo e quantitativo, realizado em 41 farmácias privadas da cidade de Nampula, entre os meses de Setembro e Outubro de 2019. A Cidade de Nampula é dividida geograficamente em 7 postos administrativos e localiza-se na Provincia de Nampula em Moçambique.

O número de Farmácias existentes na cidade de Nampula foi obtido através da direção distrital de saúde que forneceu a lista das farmácias de serviço entre setembro e Outubro de 2019.

Participaram deste estudo todas as Farmácias privadas e operacionais da cidade de Nampula. Foram excuidas do estudo as 4 Farmácias Privadas cujos resposáveis não aceitaram assinar o termo de consentimento informado bem com as farmácias que não constem na lista de escala de farmácias de serviço elaborada pela Direcção Distrital de Saúde da cidade de Nampula, para os meses de Setembro e Outubro de 2019.

\section{MÉTODO DE COLETA DE DADOS}

Para a aplicação do questionário foram treinados durante um mês os estundantes a frequentarem o IV ano do curso de licenciatura em optometria da Universidade Lúrio em Moçambique em 2019.

Ao responsável pelo estabelecimento lhes foi pedido que respondessem a um questionário semi-estruturado com 23 itens correspondentes aos nomes dos medicamentos usados para o tratamento das diferentes classificações e níveis de gravidade da conjuntivite alérgica.

A metodologia padronizada desenvolvida pelo OMS e Acção Internacional para Saúde (Health Action international - HAl) foi aplicada para a realização do estudo. A validação da metodologia WHO / HAl foi reportada em vários estudos anteriormente. Uma ferramenta de coleta de dados foi aplicada para coletar informações de disponibilidade de medicamentos para o tratamento de conjuntivite alérgica no periodo entre Setembro e outubro de 2019 (20).

Para garantir a qualidade dos dados, cada um dos Questionários foi verificado no final de cada dia de trabalho de campo e fez-se o acompanhamento de dados incompletos, errôneos ou ilegíveis. Uma vez concluída a coleta de dados, os Questionários de coleta de dados verificados foram enviados para a pesquisador principal. 


\section{SELECÇÃO DOS MEDICAMENTOS}

Como está especificado no Formulário Nacional de Medicamentos de Moçambique e na secção de oftalmologia da lista de medicamentos essencias elaborado pela OMS, essa lista que destaca alguns dos medicamentos de uso comum no tratamento da conjuntivites alérgica em paises de baixa e média renda foi adoptada para este estudo. Para além destes medicamentos, também foram adicionados medicamentos para o mesmo efeito com o apoio de dois (2) manuais: "office and emergency Room Diagnosis and treatment of eye disease" e "The Massachusetts eye and ear infirmary" (21-24).

Para a composição do Questionário foram seleccionados, Anti-histaminico, Estabilizadores de mastócitos, medicamentos de efeito combinado - Estabilizadores De Mastócitos E Anti-Histaminicos, Anti-Inflamatórios Não Esteroides, Corticosteroides, Vasoconstritor e Lágrimas artificiais.

Figura 2 - Medicamento Ocular para tratamento da conjuntivite alergica (Cetotifeno 0,125mg/0,5mL)

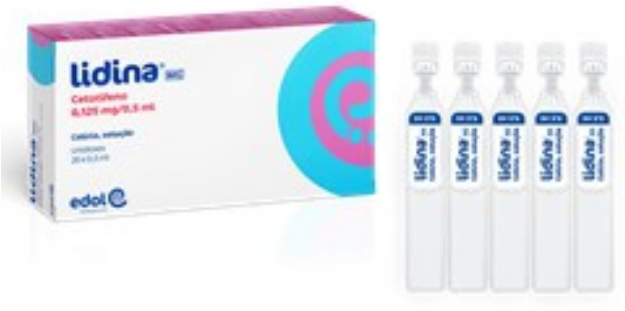

Fonte: Laboratório Edol (2019)

Figura 3 - Ficha para coleta e avaliação da disponibilidade de medicamentos tópicos e oculares para o tratamento da conjuntivite alérgica nas farmácias privadas da cidade de Nampula - Moçambique, 2019.

\begin{tabular}{|c|c|c|}
\hline \multicolumn{3}{|c|}{ LOCALIZAÇÃO DA FARMÁCIA } \\
\hline \multicolumn{3}{|l|}{ Bairro/Avenida: } \\
\hline Grupo do Medicamento & $\begin{array}{l}\text { Nome e Capacidade do } \\
\text { Frasco do Medicamento }\end{array}$ & $\begin{array}{l}\text { Disponibilidade "SIM" ou } \\
\text { "NÃO" }\end{array}$ \\
\hline \multirow{2}{*}{ Anti - Histaminico } & Emedastina, $5 \mathrm{~mL}$ & \\
\hline & Levocabastina, $4 \mathrm{~mL}$ & \\
\hline \multirow{4}{*}{ Estabilizadores de Mastócitos } & Pemirolast, $10 \mathrm{~mL}$ & \\
\hline & Nedocromil, $5 \mathrm{~mL}$ & \\
\hline & Lodoxamina, $5 \mathrm{~mL}$ & \\
\hline & Cromoglicato de sóio, $5 \mathrm{~mL}$ & \\
\hline \multirow{3}{*}{$\begin{array}{l}\text { Combinação de estabilizadores de } \\
\text { Mastócitos e Anti - Histaminicos }\end{array}$} & Azelastina, $6 \mathrm{~mL}$ & \\
\hline & Olopatadina, $5 \mathrm{~mL}$ & \\
\hline & cloridarto de Epinastina, $5 \mathrm{~mL}$ & \\
\hline
\end{tabular}




\begin{tabular}{|c|c|}
\hline & $\begin{array}{l}\text { Fumarato de cetotifeno, } \\
0,5 \mathrm{~mL}\end{array}$ \\
\hline & Bepotastina, $5 \mathrm{~mL}$ \\
\hline & Alcaftadina, $3 \mathrm{~mL}$ \\
\hline & Ketorolaco, $5 \mathrm{~mL}$ \\
\hline Antı - Inflamatorıos Nao Esteroldes & Diclofenac, $5 \mathrm{~mL}$ \\
\hline & $\begin{array}{l}\text { Etabonato de Loteprednol, } \\
5 \mathrm{~mL}\end{array}$ \\
\hline Corticoesteroides & Dexametasona, $10 \mathrm{~mL}$ \\
\hline & Fluormetalona, $5 \mathrm{~mL}$ \\
\hline & Predniselona, 5mL \\
\hline & Nafazolina, $15 \mathrm{~mL}$ \\
\hline Vasoconstritor & Feniramina, $15 \mathrm{~mL}$ \\
\hline & Antazolina, $10 \mathrm{~mL}$ \\
\hline & Hipremelose, $10 \mathrm{~mL}$ \\
\hline Lágrimas Artificiais & $\begin{array}{l}\text { Hidroxipropilmetilcelulose, } \\
10 \mathrm{~mL}\end{array}$ \\
\hline Data: $\_l \_$ & \\
\hline Assinatura do inquirido (carimbo): & \\
\hline
\end{tabular}

Fonte: Pesquisadores (2019).

\section{ANÁLISE DE DADOS}

A informação foi lançada numa planilha de trabalho do programa do Microsoft excel 2010 onde foram processados e apresentados os resultados em forma das respetivas frequências absolutas e relativas para cada medicamento presente nas farmácias no momento de aplicação do questionário.

O cálculo da disponibilidade foi realizado para cada medicamento individualmente, dividindo-se o número de estabelecimentos onde o medicamento foi encontrado pelo número de estabelecimentos onde ele deveria estar disponível.

Os valores percentuais da média para cada medicamento do Questionário foi o ponto de partida para a realização de comparações sobre a disponibilidade dos medicamentos.

\section{AVALIAÇÃO DA DISPONIBILIDADE}

A disponibilidade foi avaliada como a porcentagem de farmácias onde o medicamento foi encontrado no tempo da pesquisa e como porcentagem média de disponibilidade dos medicamentos de uso tópico e ocular selecionados para o tratamento da conjuntivite alérgica. Além disso, a percentagem média de disponibilidade de todos os medicamentos da pesquisa foi calculada para cada 
farmácia. Foram estabelecidas cinco categorias para classificar a disponibilidade: ausente $(0 \%)$, muito baixa $(<30 \%)$, baixa $(30 ;<50 \%)$, razoavelmente alta $(50 ;<80 \%)$, alta $(\geq 80 \%)$.

\section{QUESTÕES ÉTICAS}

O projecto contou com a aprovação da comissão cientifica e com a devida autorização do comitê de Bioética e pesquisa da Faculdade de Ciências de Saúde (Moçambique - Nampula) pertencente a Universidade Lúrio.

Durante a realização da pesquisa não foram utilzados nenhum exame invasivo e os responsáveis por cada farmácia foram esclarecidos sobre os objetivos e a metodologia da pesquisa e aqueles que concordaram em participar assinaram um termo de consentimento livre e esclarecido autorizando a coleta de dados.

Os entrevistadores forneceram às farmácias documentação escrita descrevendo os requisitos para participação no estudo. Ficou claro que eles poderiam se recusar a participar ou encerrar a participação a qualquer momento. $O$ consentimento escrito dos participantes do estudo foi obtido antes da coleta de dados.

\section{RESULTADOS}

Num total de 45 farmácia inscritos na cidade de Nampula, foram avaliadas 41 farmácias, destes a disponibilidade foi de $87,8 \%$, correspondente a 36 estabelecimentos com pelo menos um (1) medicamento disponível para o tratamento de conjuntivites alérgicas oculares (Tabela 1).

Tabela 1 - Número de farmácias com pelo menos um medicamento disponível

\begin{tabular}{|c|c|c|}
\hline DISPONIBILIDADE & $\mathrm{n}^{*}$ & $\%$ \\
\hline Presente & 36 & 87,8 \\
\hline Ausente & 5 & 12,2 \\
\hline Total & 45 & 100 \\
\hline
\end{tabular}

Fonte: Pesquisadores (2019). $\mathrm{n}^{*}$ número das farmácias

Os 23 medicamentos selecionados foram distribuídos em 7 grupos. Os corticosteroides são os mais disponíveis com 82,9\%, encontrados em 34 farmácias, depois os estabilizadores de mastócitos com 46,3\%, encontrados em 19 farmácias. Os anti-histamínicos não foram encontrados em nenhuma farmácia, considerando se indisponível nas farmácias da cidade de Nampula (Gráfico 1 e Gráfico 2). 
Gráfico 1 - Disponibilidade dos Medicamentos nas Farmácias por grupos

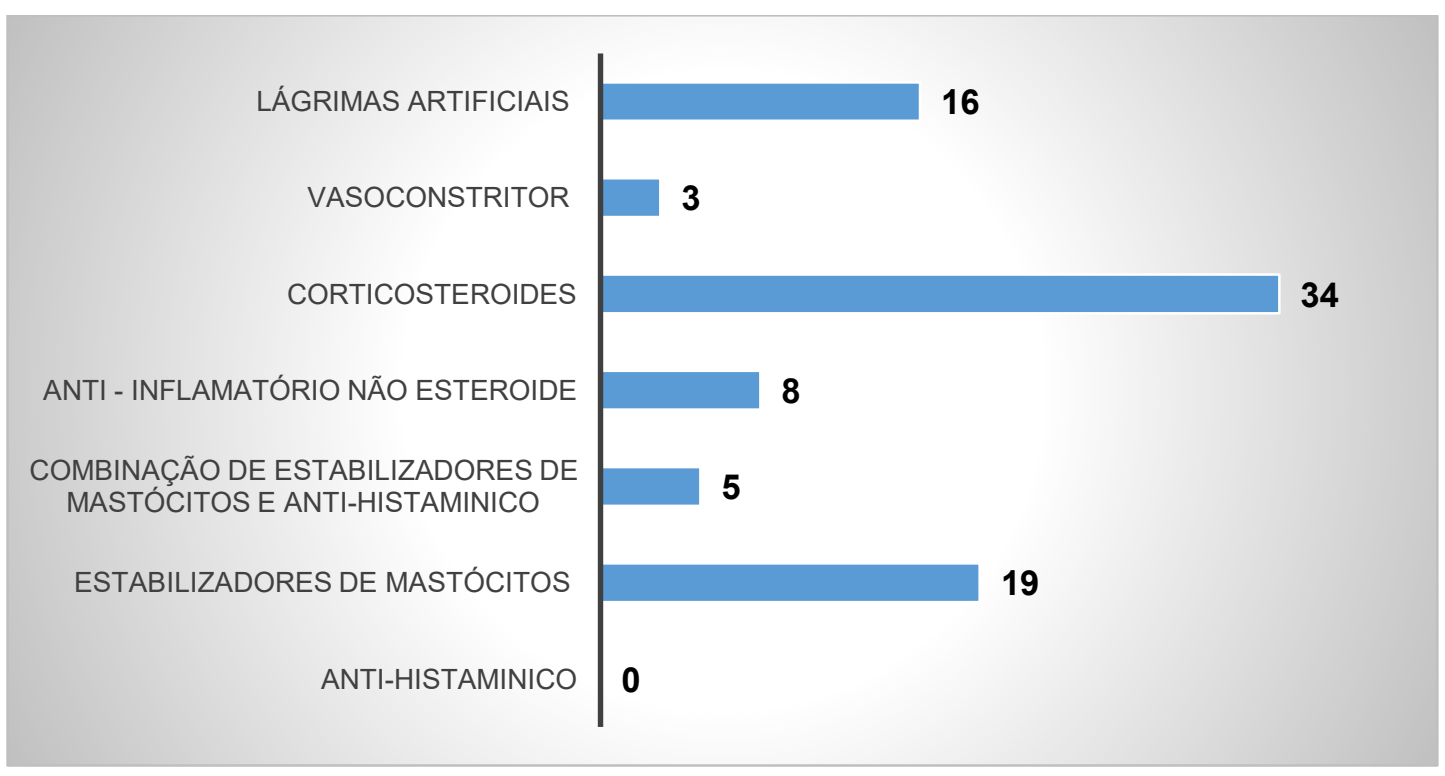

Fonte: Pesquisadores (2019)

Gráfico 2 - Número de Medicamentos diversificados por cada Grupo

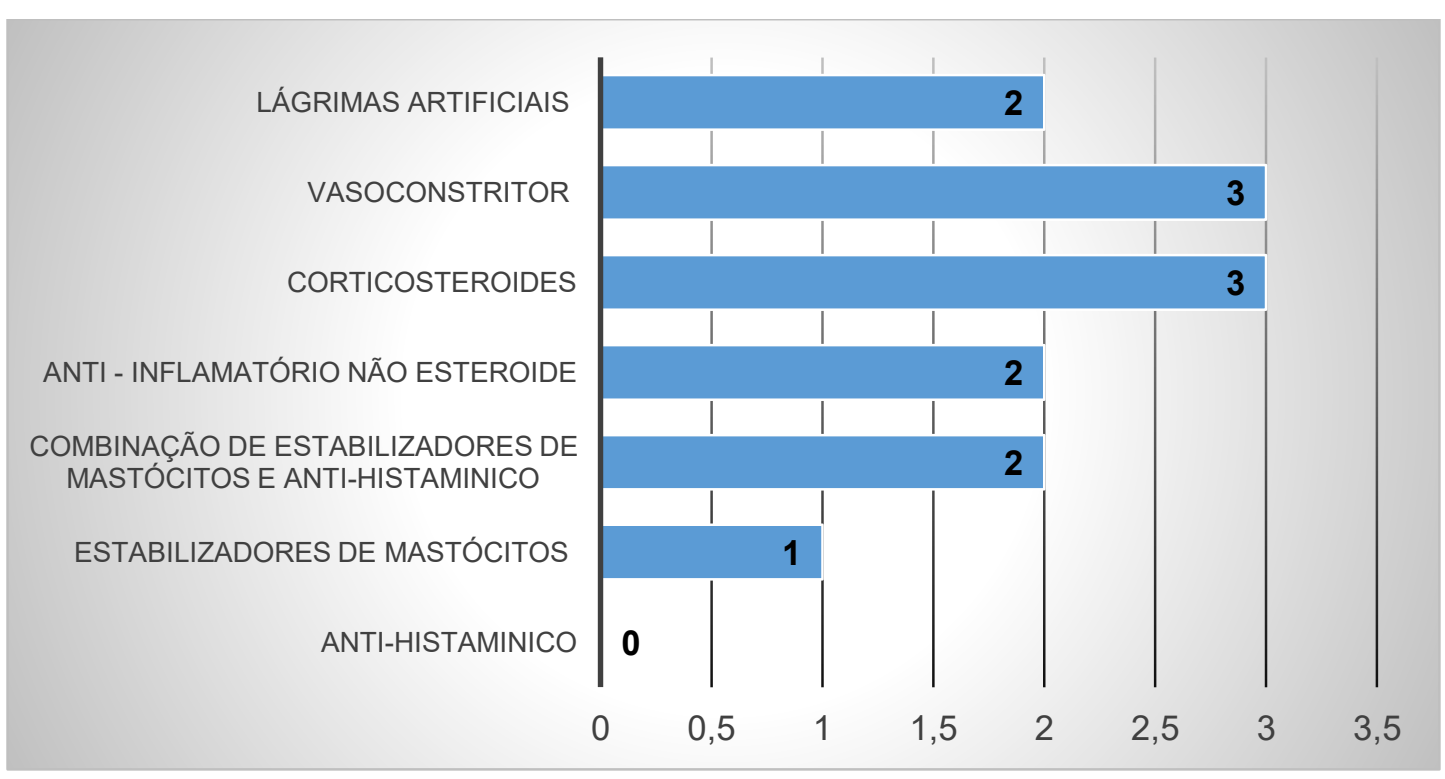

Fonte: Pesquisadores (2019)

$\mathrm{Na}$ análise da Tabela 2, dos 23 medicamentos selecionados, apenas 13 é que estão disponíveis, sendo a prednisolona o medicamento mais encontrada com $82,9 \%$ seguido de dexametasosa, Cromoglicato de Sódio e lágrimas artificiais (hipromelose) com $68,3 \%, 46,3 \%$ e $39,0 \%$ respectivamente.

De acordo com a Tabela 3, A disponibilidade média dos medicamentos é relativamente alta $(58,52 \%)$. 
Tabela 2 - Disponibilidade de cada medicamento nas farmácias

\begin{tabular}{l|c|c}
\multicolumn{1}{c|}{ NOME DO MEDICAMENTO } & \multicolumn{2}{c}{ DISPONIBILIDADE } \\
\cline { 2 - 3 } & $\mathbf{n}^{*}$ & $\%$ \\
\hline Emedastina 5mL & 0 & 0 \\
Levocabastina 4mL & 0 & 0 \\
Pemirolast $10 \mathrm{~mL}$ & 0 & 0 \\
Nedocromil 5 mL & 0 & 0 \\
Lodoxamina 5mL & 0 & 0 \\
Etabenato de loteprodnol 5mL & 0 & 0 \\
Azelastina 6mL & 0 & 0 \\
Bepotastina 5mL & 0 & 0 \\
Alcaftadina 3mL & 0 & 0 \\
cloridrato de Epinastina 5mL & 0 & 0 \\
Antazolina 10mL & 1 & 2,4 \\
Feniramina 15mL & 1 & 2,4 \\
Fluorometolona 5mL & 1 & 2,4 \\
Ketorolaco 5mL & 1 & 2,4 \\
Nafazolina 15mL & 3 & 7,3 \\
Olopatadina 5mL & 4 & 9,8 \\
Fumarato de Cetotifeno 0,5mL & 5 & 12,2 \\
Diclofenac 5mL & 8 & 19,5 \\
Hidroxymetilpropilcelulose 10mL & 9 & 22,9 \\
Hipromelose 10mL & 16 & 39,0 \\
Cromoglicato de Sódio 5mL & 19 & 46,3 \\
Dexametasona 10mL & 28 & 68,3 \\
Prednisolona 5mL & 34 & 82,9 \\
\hline
\end{tabular}

Fonte: Pesquisadores (2019)

Tabela 3 - Categorias de acordo com a disponibilidade dos medicamentos nas farmácias privadas da cidade de Nampula

\begin{tabular}{|c|c|c|c|}
\hline \multicolumn{2}{|c|}{ Categoria de Disponibilidade } & No. & \\
\hline $0 \%$ & Ausente & 10 & $43.48 \%$ \\
\hline$>0 \%-<30 \%$ & Muito Baixa & 9 & $39.13 \%$ \\
\hline $30 \%-<50 \%$ & Baixa & 2 & $8.70 \%$ \\
\hline $50 \%-<80 \%$ & Relativamente Alta & 1 & $4.35 \%$ \\
\hline$\geq 80 \%$ & Alta & 1 & $4.35 \%$ \\
\hline \multicolumn{4}{|c|}{ Disponibilidade Média de todos medicamentos Avaliados } \\
\hline $50 \%-<80 \%$ & Relativamente Alta & 13 & $56.52 \%$ \\
\hline
\end{tabular}

Fonte: Pesquisadores (2019).

\section{DISCUSSÃO}

Neste estudo a maior parte dos medicamentos que foram avaliados, mostraram-se disponíveis nas farmácias privadas da cidade de Nampula. Aproximadamente um terço da população mundial tem acesso a medicamentos, pelos elevados preços, sendo que essa proporção aumenta para $50 \%$ em países em desenvolvimento ${ }^{(25)}$. 
O facto de, na sua maioria, a disponibilidade dos medicamentos para o tratamento da conjuntivite alérgica variar de baixa a ausente, deve contribuir para desigualidades no acesso a medicamentos pela inflacção de seus preços. $\mathrm{O}$ acesso equitativo a medicamentos essenciais faz parte do cumprimento do direito ao mais alto padrão de saúde possível. No entanto, os altos preços dos medicamentos tornam os medicamentos inacessíveis para muitas pessoas (26). A disponibilidade de medicamentos nos países em desenvolvimento ainda precisa ser melhorada, aumentando o acesso a medicamentos e controlando os preços, usando políticas governamentais apropriadas ${ }^{(27)}$. Por outro lado, investir em inovação e na produção de novos medicamentos sem que os mesmos sejam acessíveis é favorecer o comércio, o lucro desmedido e, em certas ocasiões, até extorsivo, em detrimento da saúde e dos princípios que regem o direito à vida com dignidade (28).

\section{LIMITAÇÕES DO ESTUDO}

O estudo tem várias limitações que são consistentes com pesquisas anteriores. A metodologia da OMS / HAI não avalia as alternativas terapêuticas ou alternativas posologicas. Os resultados do estudo refletem o status da disponibilidade com base no dia da coleta de dados. Eles não necessariamente refletem a disponibilidade mensal ou anual dos medicamentos para o tratamento da conjuntivite alérgica a nível nacional. Além disso, a comparação do custo médio dos medicamentos, o acesso a esses medicamentos nas farmácias privadas não foram ilustrados neste estudo, nem avaliadas.

A aplicação do questionário para a avaliação da disponibilidade do medicamentos aqui abordada, não permite tirar conclusões sobre o custo médio e o acesso nas farmácias privadas. A falta de estudos sobre a disponibillidade de medicamentos oculares tanto a nível nacional, regional assim como no nível internacional, limita a realização de comparações com os resultados deste estudo.

Por outro lado, o estudo utilizou uma metodologia anteriormente validada usando uma maneira padronizada de avaliar a disponibilidade dos medicamentos para o tratamento da conjuntivite alérgica ${ }^{(29)}$.

Além disso, o treinamento e o uso de múltiplos pontos de controle melhoraram a qualidade da coleta de dados, lançamento e interpretação de dados.

\section{CONCLUSÃO}

Depois da realização do estudo conclui-se que a disponibilidade média de medicamentos para o tratamento da conjuntivite alérgica nas farmácias da cidade de Nampula é relativamente alta, existe um número variado e significativo de medicamentos para o tratamento da conjuntive alérgica, onde os corticoesteroides e 
os estabilizadores de mastócitos são representativos, em contra-partida os antihistaminicos não existem.

Embora existam ainda dificuldades na existência de medicamentos para o tratamento de conjuntivite alérgica nas farmácias da cidade de Nampula, alguns grupos de medicamentos apresentam disponibilidade alta e outros baixa. Torna-se, por isso, importante orientar as farmácias a cobertura de medicamentos de diferentes linhas de tratamento para a conjuntivite alérgica.

Esta evidente, que são necessários mais estudos de intervenção, junto das farmácias e dos utentes, que possibilite o entendimento não apenas da disponibilidade mas do acesso e da capacidade de aquisição dos médicamentos nas farmácias privadas, obedecendo obviamente as normas da OMS para o efeito.

\section{REFERÊNCIAS}

1. Instituto nacional de Estatistica. IV recenseamento geral da população e habitação 2017; Resultados definitivos moçambique Maputo. Abril; 2019.

2. Mozambique GDP (Real Growth Rate). Economy, 2019. Disponivel em: https://www.indexmundi.com/mozambique/gdp real growth rate.html.

3. Resnikoff S, Bourne R. World blindness and visual impairment: despite many successes, the problem is growing. Community eye health journal, 2017; 30(100):71-73.

4. Bright T, Kuper H, Macleod D, Musendo D, Irunga P, Yip JLY. Population need for primary eye care in Rwanda: A national survey. PLoS ONE 2018; 13(5): e0193817. https://doi.org/ 10.1371/journal.pone.0193817.

5. Lee L, D’Esposito F, Garap J, Wabulembo G, Koim SP, Keys D, Cama AT, Limburg $\mathrm{H}$ e Burnett A. Rapid assessment of avoidable blindness in Papua New Guinea: a nationwide survey. Br J Ophthalmol, 2019; 103:338-342.

6. Mello-Bochi, CB. Modelo experimental de conjuntivite alérgica: efeito do tratamento farmacológico com a proteína anti-inflamatória galectina - 1. 2014. 106 f. Dissertação (mestrado) - Universidade Estadual Paulista Julio de Mesquita Filho, Instituto de Biociências, Letras e Ciências Exatas; 2014. Disponível em: $<$ http://hdl.handle.net/11449/122179>.

7. Katibeh M, Pakravan M, Yaseri M, Pakbin M, Soleimanizad R. Prevalence and causes of visual impairment and blindness in central Iran. The Yazd eye study. J Ophthalmic Vis Res 2015; 10:279-85.

8. Torres JP, Santos V. Conhecendo a deficiência visual em seus aspectos legais, históricos e educacionais. Educação, Batatais 2015; 5(2):33-52. 
9. Geraldini M, Chong Neto MJ, Riedi CA, Rosário NA. Epidemiology of ocular allergy and co-morbidities in adolescents. J Pediatr (Rio J). 2013; 89:354-60.

10. Malu KN. Vernal keratoconjunctivitis in Jos, North-Central Nigeria: A hospitalbased study. Sahel Med J 2014; 17:65-70.

11. Sandrin LNA, Santo RM. Perspectivas no tratamento da alergia ocular: revisão das principais estratégias terapêuticas. Rev. bras. oftalmol. 2015; 74(5): 319324.

12. Álvares $\mathrm{J}$ et al. Access to medicines by patients of the primary health care in the Brazilian Unified Health System. Revista de Saúde Pública [online]. 2017, v. 51, suppl 2 [Acesso em 25 Março 2020], 20s. DOI: https://doi.org/10.11606/S1518$\underline{8787.2017051007139 .}$.

13. Matine J. Falta de medicamento nos hospitais públicos associada a falta de responsabilização dos gestores do Sistema Nacional de Saúde. CIP, Serviço De Partilha de Informação, ed 6; 2015.

14. Rocha SA, Bocchi SCM e Godoy MF. Acesso aos cuidados primários de saúde: revisão integrativa. Physis: Revista de Saúde Coletiva [online]. 2016, v. 26, n. 1 [Acesso em 25 Março 2020], pp. 87-111. DOI: https://doi.org/10.1590/S010373312016000100007 .

15. Mendes LV et al. Disponibilidade de medicamentos nas unidades básicas de saúde e fatores relacionados: uma abordagem transversal. Saúde em Debate [online]. 2014, v. 38, n. spe [Acesso em 25 Março 2020], p. 109-123. DOI: https://doi.org/10.5935/0103-1104.2014S009.

16. Sousa TM, Guedes RC, Valadão FA e Brum CA. Análise da disponibilidade de medicamentos genéricos em farmácias e drogarias no município de lpatinga MG. Revista Brasileira de Farmácia, 2011;91(2):89-95.

17. Bourne RR, Flaxman SR, Braithwaite T, Cicinelli MV, Das A, Jonas JB et al. Magnitude, temporal trends, and projections of the global prevalence of blindness and distance and near vision impairment: a systematic review and meta-analysis. The Lancet Global Health, 2017;5(9), 888-897.

18. Attridge CJ, Preker AS. Improving Access to Medicines in Developing Countries: Application of New Institutional Economics to the Analysis of Manufacturing and Distribution Issues. Health, Nutrition and Population (HNP) discussion paper; World Bank, Washington, DC. @ C World Bank. 2005. Disponível em: https://openknowledge.worldbank.org/handle/10986/13668

19. Sterckx S. Patents and access to drugs in developing countries: an ethical analysis. Developing World Bioethics, 2004; 4(1):58-75.

20. Dorj G, Sunderland B, Sanjjav T \& Gendenragchaa B. Availability, affordability and costs of pediatric medicines in Mongolia. BMC pediatrics, 2018; 18(1), 149. DOI: https://doi.org/10.1186/s12887-018-1123-x. 
21. MISAU - Departamento Farmacêutico. Lista de Medicamentos de Essencias; 2017. Disponivel em: https://www.afro.who.int/sites/default/files/2018.

22. MISAU. Formulário Nacional de Medicamentos de Moçambique; 2007. Disponivel em: https://augustobene.com/formulario-nacional-de-medicamentosde-mocambique-2007.

23. Ehlers JP, Shah CP. The Wills eye manual: office and emergency room diagnosis and treatment of eye disease. 5th ed. Philadelphia. Lippincott Williams \& Wilkins, 2008.

24. Friedman NJ, Kaiser PK, Pineda II R. The Massachusetts eye and ear infirmary: Illustrated Manual of ophthalmology. 3th Ed. Saunders Elsevier.

25. Ruggie J. Report of the Special Representative of the Secretary-General on the Issue of Human Rights and Transnational Corporations and other Business Enterprises: Guiding Principles on Business and Human Rights: Implementing the United Nations 'Protect, Respect and Remedy' Framework. Netherlands Quarterly of Human Rights, 2011; 29(2), 224-253.

26. Fang Y, Wagner AK, Yang S, Jiang M, Zhang F e Ross-Degnan D. Access to affordable medicines after health reform: evidence from two cross-sectional surveys in Shaanxi Province, western China. The Lancet Global Health, 2013; 1(4): e174-e175.

27. Latifah E, Kristina SA, Satibi SS. Overview of Drug Availability and Influencing Factors in Several Low, Lower and Upper- Middle Countries: A Systematic Review. Sys Rev Pharm. 2019;10(1):67-72.

28. Bermudez JAZ. Acesso a medicamentos: impasse entre a saúde e o comércio! Cad. Saúde Pública 2017; 33(9):e00123117.

29. World Health Organization \& Health Action International. Measuring medicine prices, availability, affordability and price components, 2nd edition. World Health Organization; 2008. Disponível em: https://apps.who.int/iris/handle/10665/70013.

\section{AGRADECIMENTOS}

Gostariamos de agradecer as farmácias pela colaboração ao aceitarem fazer parte deste estudo. Aos estundantes do IV do curso de licenciatura em Optometria pela dedicação, empenho e entrega no processo de colecta de dados, nosso muito obrigado.

Artigo recebido em: $17 / 04 / 2020$

Artigo aprovado em: 14/12/2020

Artigo publicado em: 18/01/2021 